\title{
We Can Do It for Free!
}

\author{
Using Freeware for Online Patron Engagement \\ Karin Suni and Christopher A. Brown
}

In the early weeks of the pandemic, the Special Collections Division of the Free Library of Philadelphia (https://freelibrary.org/) responded to the library's call for fun and interactive online engagement. Initially staff members released games and Buzzfeed-inspired lists via various social media accounts to amuse patrons, distract from the lockdown, and provide educational programming. As the list of activities grew, we realized this content needed a more substantial home; the return on investment of time for the development and production of an online game to be released once on social media was not sufficient. Activities and passive programming that took hours to create could easily fall victim to social media's algorithms and be quickly buried in a patron's feed. The Free Library's official blog was an insufficient option because it promoted all library programming, and our goal was to highlight the value of our division and the materials housed within it. We resolved these issues by creating an online repository solely with freeware systems (https://bit.ly/FunWithFLPSpecColl). The repository provides a stable landing page wherein the Special Collections Division content builds meaningful connections with patrons of all ages. This model can be readily adapted and is a valuable tool for library workers promoting their own online engagement.

\section{REPOSITORY FRAMEWORK}

It was clear that our division could not add to the burden of an overworked IT staff by requesting support for digital engagement. We needed to seek external alternatives that would interest patrons and could be managed with limited training. Before we began our search, we brainstormed a list of requirements:

- An inexpensive and user-friendly hosting platform

- A pleasing look and easy navigation

- The ability to be updated frequently and easily

- The flexibility to adapt and expand as our requirements change

Our search led us to the Google suite of products, specifically Google Sites and Google Drawings. Google Sites and Google Drawings integrated perfectly with each other, and we appreciated their usability and relative simplicity. Once we selected the software, we knew we needed a list of best practices to guide the repository's creation:

- To establish a visual connection with our official website, the repository would primarily use the Free Library's branded color scheme.

- All thumbnails created would be square, allowing us to reuse the image as promotional material on different social media accounts.

Karin Suni (sunik@freelibrary.org) is Curator, Theatre Collection, the Free Library of Philadelphia. Christopher A. Brown (brownc@freelibrary.org) is Curator, Children's Literature Research Collection, the Free Library of Philadelphia. (C) 2021.

"Public Libraries Leading the Way" is a regular column spotlighting technology in public libraries. 
- All members of the division can create content, but the ability to update and edit the repository would remain limited to ensure consistency.

These guidelines have proven effective. The color scheme and thumbnail rules formed a framework wherein we could work productively without "reinventing the wheel." Limiting administrative abilities has allowed us to maintain a controlled vocabulary within the repository, better unifying the content.

\section{REPOSITORY SOFTWARE}

The Google Suite, specifically Google Sites, is advantageous for library workers looking to create professional-looking content quickly. It is free with a Google account and built-in templates allow users to build a fully functional website within a few hours with little-to-no design experience. As with all freeware, Google Sites has quirks. The foremost is that while there are options for customization, these options are finite. There are a limited number of layout, header, and font designs meaning that anyone using the software must temper their vision to fit within the confines of the program.

Google Drawings is far more flexible, in part because it is a much simpler program. Users familiar with software like PowerPoint or MS Paint have the ability to design images for headers, thumbnails, etc. Two drawbacks we encountered with this freeware are the restrictions on image upload size (a consideration for our division given the archival files used in our digital collections) and the limited ability to create word art. For our division, the advantages of these software products outweigh their limitations.

\section{CONTENT FRAMEWORK}

The repository houses programming devised primarily with freeware. An early discovery was a suite of activities from Flippity (https://www.flippity.net). Designed for educational use, Flippity provides templates for a variety of online activities including memory games, matching games, and board games. Our primary focus has been on the first two, although we continue to explore new aspects of this suite as templates are added. Flippity works with Google Sheets and can integrate images from Google Drawings.

Jigsaw Planet (https://jigsawplanet.net/) has been used extensively by libraries and museums during the pandemic. It allows creators to easily turn images into puzzles that are played online, either on the site itself or through embedding the puzzle. The site allows registered users to access leaderboards, and it allows creators to track how many times puzzles have been played. In addition to the ease of use, the major benefit of Jigsaw Planet is that the patron can customize their experience by changing the number of pieces to fit their preferred level of difficulty.

The desire for audio and video content has surged over the last several months, and we have sought to meet that need through the use of a variety of software. In regard to video, YouTube is not a new tool, but the majority of our pre-pandemic programs were not filmed. With the shift to Crowdcast and Zoom, we now have a library of online lectures and other events that have been uploaded to YouTube and can be viewed repeatedly and at any time. With a dedicated home for this content, we have been inspired to seek out older videos of Special Collections programming across multiple channels and link them to the repository. 
One of the newest additions to our offerings has been the podcast Story Search From Special Collections (http://bit.ly/flpstorysearch), which explores stories based on, inspired by, or connected to material artifacts. The podcast is recorded and edited using Zencastr and Audacity and is posted on Anchor, which also distributes it to major listening apps.

In recent weeks, our division has added images, blog posts, and additional content for current and past exhibitions. This is the first formal exhibition compilation since the Special Collections Division began in 2015, and we are delighted that it is available for the public to explore. The material is arranged using templates and tools available in Google Sites, allowing patrons to view image carousels, exhibition tags, and past programs. The inclusion of this material marks a shift away from the repository functioning as a response to the need for pandemic-related content to a living history of our division and our work promoting the Special Collections of the Free Library.

\section{ACCESSIBILITY}

Accessibility and equity of access lie at the core of library service. Sadly, we were not initially focused on this point, and our content was not fully accessible, e.g., text was presented in thumbnails only which limited the use of screen readers to relay information. As the content expanded, we sought to make the space as inclusive as the freeware limits allowed. Alternative text was added to images and information was not limited within thumbnails. This is an ongoing process, but one that is necessary to reach as many patrons as possible.

\section{ANALYTICS}

Site visits and other statistics for a library's online presence are always important, but especially so during the pandemic when restricted physical access has driven more patrons to online resources. Our plan for capturing this information was two-pronged. First, we used bit.ly to create customized, trackable links for our content. These are used within the repository and on social media and in other online promotions. This has proven to increase repository traffic while providing information on how patrons discover our content. The statistics generated from bit.ly are only available for 30 days for free accounts, albeit in a rolling 30 -day window. Knowing this, we transcribe the statistics monthly into a spreadsheet to maintain a consistent account of patron access.

Our second prong is Google Analytics, a freeware option that only tracks data within the repository. Google Analytics connects a single Google account to Google Sites, but the integration is seamless and the data remains available indefinitely. This provides a visual breakdown of statistics, including maps and graphs that are easily shared with other stakeholders. By using both tools we are able to surmise who is visiting the repository, where they are finding the links, and which sections are popular with our patrons.

\section{CONCLUSION}

The Special Collections repository was created in response to a growing need for online patron engagement during the early weeks of the pandemic. Our division strove to engage the public with fun, educational programming and activities primarily using freeware. This has proven to be successful with the general public and members of our division. The statistics from the site have both informed content creation and engendered a better appreciation for the repository from our administration. As we move forward, the repository is evolving into a comprehensive collection of what the Special Collection Division does and how we meet the need for patron engagement 
online and in person. It is a framework that can be used by library workers across a multitude of areas and specialties, housing activities from story times and passive programming to book clubs and lectures. 